\title{
Comparison between 3D-Transvaginal Ultrasound and Hysteroscopy in Detecting Uterine Cavity Abnormalities
}

\author{
Ahmed Hassan El Tagy, Ahmed Osama Abd El Motaal, Mahmoud Awaly* \\ Department Of gynecology and obstetrics, Faculty of Medicine, Al-Azhar University, Cairo, Egypt \\ *Corresponding author: Mahmoud Awaly, Mobile:01006213906,E-mail:Mahmoud_awaly@hotmail.com
}

\section{Abstract}

Background: unsuspected uterine cavity abnormalities, such as endometrial polyps, small submucous myomas, adhesions, and uterine septum may cause different gynecological complaints such as bleeding and negatively impact chances of success in ART by interfering with implantation or causing spontaneous abortion. Therefore, exclusion of intrauterine pathology is an important step before starting ART and treatment of any discovered lesion may improve ART outcome. The uterine cavity can be evaluated by hysterosalpingography (HSG), transvaginal ultrasound (TVS), sonohysterography (SHG), and hysteroscopy. Hysteroscopy is the gold standard for investi-gation of the uterine cavity, particularly when pathology is suspected.

Objective: to assess diagnostic accuracy of three dimensional transvaginal ultrasound (3D-TVS) compared with hysteroscopy in detecting uterine cavity abnormalities.

Study design: this prospective observational cross-sectional study was conducted during the January 2018 to June 2018. One hundred women with different uterine pathologies were enrolled. In the mid to late follicular phase of each subject's menstrual cycle, three D transvaginal ultrasound and hysteroscopy were performed in each patient. Hysteroscopy is widely considered to be the gold standard method for investigation of the uterine cavity. Uterine cavity characteristics and abnormalities were recorded. Diagnostic accuracy, sensitivity, specificity, positive predictive value, negative predictive value, and positive and negative likelihood ratios were evaluated.

Results: hysteroscopy was successfully performed in all subjects. Hysteroscopy diagnosed pathological findings in 100 of 100 cases (100\%). There were 50 endometrial polyps, 13 submucous myomas, 29 septate uterus, and 8 intrauterine adhesions. Three-dimensional transvaginal ultrasound in comparison with hysteroscopy had $85 \%$ diagnostic accuracy, $68.2 \%$ sensitivity, $91.5 \%$ specificity, $79 \%$ positive predictive value, and $86 \%$ negative predictive value. The positive and negative likelihood ratios were 8.01 and 0.3 , respectively. 3D-TVS successfully detected every case of submucous myoma. For detection of endometrial polyps, 3D-TVS had $80.0 \%$ sensitivity, $100.0 \%$ specificity, and $90 \%$ diagnostic accuracy. For detection of septate uterus, 3D-TVS had $93.10 \%$ sensitivity, $100.0 \%$ specificity, and $98 \%$ diagnostic accuracy.

Conclusion: three D-TVS demonstrated $84.1 \%$ diagnostic accuracy for detecting uterine cavity abnormalities in infertile women. A significant percentage of patients had evidence of uterine cavity pathology. Hysteroscopy is, therefore, recommended for accurate detection and diagnosis of uterine cavity lesion.

Keywords: Uterine cavity abnormality, three D transvaginal ultrasound, Hysteroscopy, Infertility.

\section{Introduction:}

The uterine cavity can be evaluated by hysterosalpingography (HSG), transvaginal ultrasound (TVS), sonohysterography (SHG) and hysteroscopy. Hysteroscopy is the gold standard for investigation of the uterine cavity, particularly when pathology is suspected. Advantages of hysteroscopy are that it permits direct visualization of the uterine cavity, showing characteristics of lesions, including nature, size, shape, location, and vascular pattern ${ }^{(1)}$.

Unsuspected uterine cavity abnormalities, such as endometrial polyps, small submucous myomas, adhesions, and uterine septum may be symptomatic and cause abnormal uterine bleeding or oligomenorrhea or negatively impact chances of success in ART by interfering with implantation or causing spontaneous abortion ${ }^{(2)}$.

Intrauterine pathologies were found in $11-40 \%$ of infertile patients ${ }^{(3)}$. Therefore, exclusion of intrauterine pathology is an important step before starting ART and treatment of any discovered lesion may improve ART outcome $^{(\mathbf{1})}$.

Reports vary regarding the diagnostic accuracy of 3D-TVS. 3D-TVS was reported to have $41.3-81.5 \%$ sensitivity and $94.6-98.7 \%$ specificity ${ }^{(4)}$.

Three D-TVS may have limitations in detection of submucous fibroids in the presence of 
multiple fibroids, intrauterine adhesion, distinguishing hyperplastic endometrium from polyp, and distinguishing arcuate from septate uterus ${ }^{(\mathbf{1})}$.

3D-TVS examines three planes simultaneously, producing a $3 \mathrm{D}$ rendering and reconstruction of the coronal plane, both of which are not visible in 2D-US ${ }^{(5)}$.

Three D images can be developed from data obtained with a single sweep of the US beam across the involved organ; a factor that may decrease interobserver variability ${ }^{(6)}$.

The role of three D-TVS in evaluation of uterine cavity lesion in infertile women remains unclear. Three D-TVS may successfully and adequately overcome the limitations of 2D ultrasound and may improve diagnostic accuracy in the detection of uterine cavity abnormalities ${ }^{(7)}$. Hysteroscopy is the gold standard for investigation of the uterine cavity, particularly when pathology is suspected. Advantages of hysteroscopy are that it permits direct visualization of the uterine cavity, showing characteristics of lesions, including nature, size, shape, location, and vascular pattern ${ }^{(\mathbf{1})}$. Although hysteroscopy has very few associated complications, perforation, cervical laceration, bleeding, and vasovagal reaction may occur ${ }^{(8)}$. Hysteroscopy may also achieve only limited access in cases of cervical stenosis (8).

The aim of this study was to determine the diagnostic accuracy of 3D-TVS compared with hysteroscopy in detecting uterine cavity abnormalities in women with different uterine pathologies.

Patients and Methods:

This prospective observational cross-sectional study was conducted at the Department of Obstetrics and Gynecology, Faculty of Medicine, Al Azhar University, (El-Hussine) hospital.

This study was approved by the Institutional Review Board of the Faculty of Medicine, Al Azhar University. From January 2018 to June 2018, 100 women with different gynecological complaints; mainly bleeding or infertility were recruited according to the following inclusion

\section{criteria:}

1- 18-45 years of age.

2- No history of hysteroscopy.

3- No history of IVF.

4- Absence of active STDs, PID, and active vaginal bleeding.

Patients were excluded if they:

1. Are under 18 years.

2. High risk for undergoing hysteroscopy (e.g., congestive heart failure).

3. Normally fertile women.

4. Other causes of infertility:

- Peri-tubal surgeries.

- Peri-ovarian cystectomy surgeries.

- Laparoscopic ovarian drilling.

- Endometriosis.

All subjects provided written informed consent. The least calculated number of female that could be included in that study was 86 patients. A total of 100 participants were included, the calculation was based on a statistical significance level of $0.05 \%$ to yield a power of $90 \%$. Threedimensional transvaginal ultrasound was performed by a single operator (VP) using a Voluson E6 Expert 3D-US system (GE Healthcare GmbH \& Co OG, Tiefenbach, Zipf, Austria) with a RIC5- 9H transvaginal probe. Ultrasound was performed in the mid to late proliferative phase of the menstrual cycle. Sonographic technique was standardized according to the following criteria: probe frequency set at $9 \mathrm{mHz}$,

- A midsagittal view of the uterus filling $75 \%$ of the screen,

- Three-dimensional box size including the uterine fundus to the cervix,

- Sweep angle of 908,

- And 3D volume was obtained through the uterus by an automated system.

The image was then presented in a multiplanar display, showing three perpendicular planes through the volume: the sagittal, transverse, and reconstructed coronal views. By scrolling through the reconstructed coronal view, multiple images were obtained by the sonographer through the anteroposterior thickness of the endometrium, as presented in Fig. 1. 


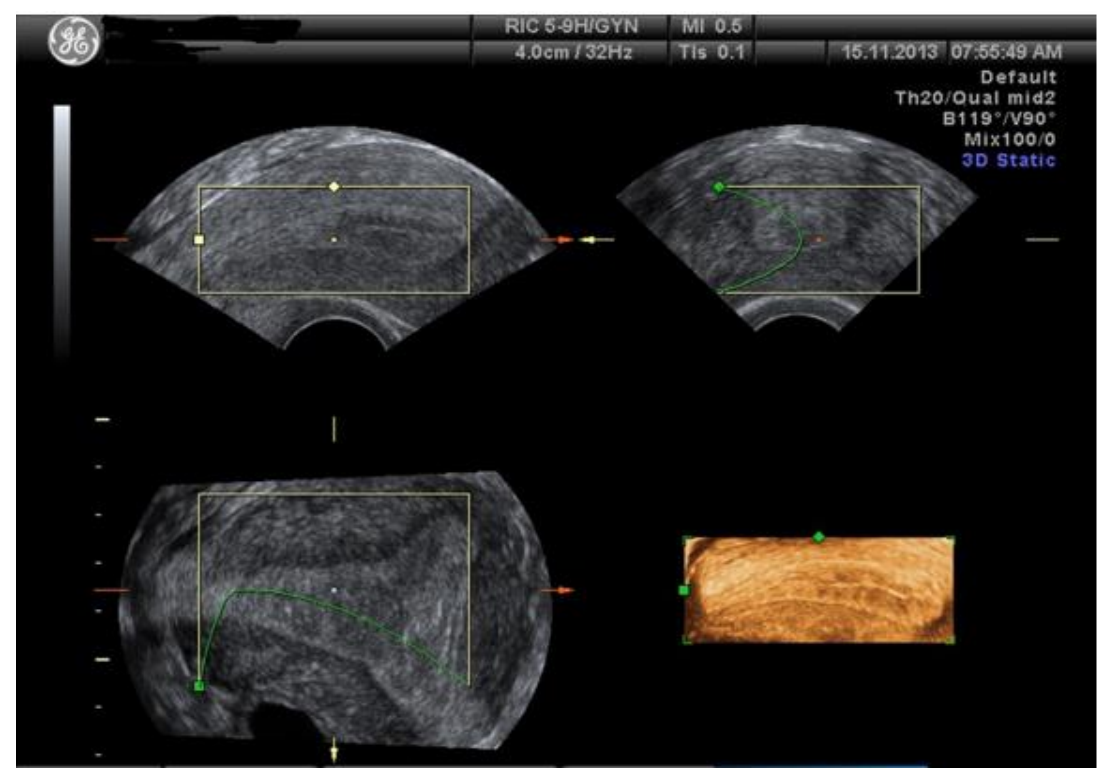

Fig (1). Multiplanar imaging of uterus from 3D-TVS.

The endometrium and surrounding myometrium were evaluated for configuration of the cavity, masses, and the relationship of masses to the endometrial cavity. To accurately assess uterine contour and shape of the endometrial cavity, careful evaluation of the perpendicular planes was performed by the sonographer to ensure that a true midcoronal view of the uterus was obtained.

Hysteroscopy was also performed. Hysteroscopy was performed by a single operator, under supervision of two expert endoscopists. General anaesthesia was given to all the patients before dilatation of the cervix and introduction of the hysteroscope.

STORZ 26120 BA hopkins II $2.9 \mathrm{~mm}$ 26120BA Bettocchi Hysteroscope is introduced into external cervical os with direct vision after cervical dilatation using Hegar dilators is performed.

Normal saline solution was used as a distention media and was instilled under the pressure of conventional blood pressure cuff.

The hysteroscope was then gently introduced through cervical canal, internal os, and then into the uterine cavity.

Upon entering the uterine cavity, a systematic inspection was performed, including the uterine cornu, tubal ostia, uterine fundus, and lateral, anterior and posterior uterine walls.

Hysteroscopy findings were documented on a case record form. Appearance of cervical canal and endometrium and presence, size, and location of structural anomalies were recorded. In case of positive findings for uterine cavity lesion, surgical management options were done on the same setting.
Sensitivity, specificity, diagnostic accuracy, positive predictive value, negative predictive value, positive likelihood ratio, and negative likelihood ratio of 3D-TVS were calculated and compared with hysteroscopic diagnosis, the gold standard for evaluation of the uterine cavity.

Diagnostic accuracy was defined as the proportion of true results (both true-positive and true-negative) in the study population, and was calculated as ([number of truepositive + number of true-negative]/total number of study population).

\section{Results:}

One hundred women completed the study. Average age of subjects was 30.38 years as shown in (Table 1). 3D transvaginal ultrasound and hysteroscopy was successfully performed in all women.

Among the studied population, 39 women complained from bleeding whereas the infertility was the main complaint of the other 61 women (Table 2). Ultrasonographic and hysteroscopic findings are shown in (Table 3 ). Hysteroscopy showed 100 cases of positive findings, including endometrial polyp in 50 patients, submucous myoma in 13 patients, 8 cases of adhesions and 13 case of uterine anomaly. 3D-TVS missed the diagnosis of ten endometrial polyps, 3 cases of adhesions and 2 cases of septate uterus but could successfully diagnose all cases of submucous myoma.

\section{Statistical analysis:}

As a clinical investigation for detection of uterine cavity abnormalities, 3D-TVS in comparison with hysteroscopy demonstrated $68.2 \%$ sensitivity, $91.5 \%$ specificity, $84.1 \%$ 
diagnostic accuracy, $79 \%$ positive predictive value (PPV), and $86 \%$ negative predictive value (NPV) (Table 4). The positive and negative likelihood ratios were 8.01 (95\% CI 3.0121.34) and 0.35 (95\% CI $0.19-0.65)$, respectively. Prevalence of uterine cavity lesion in this study was $100.0 \%$. The most common lesion was endometrial polyp (50.0\%). Prevalence of submucus myoma and uterine anomaly were $13 \%$ and $29 \%$, respectively as shown in (Table 3).

Table (1): Distribution of the studied cases according to age $(n=100)$

\begin{tabular}{|c|c|c||}
\hline Age (years) & No. & \% \\
\hline$\leq 30$ & 56 & 56.0 \\
$>30$ & 44 & 44.0 \\
\hline Min. - Max. & \multicolumn{2}{|c|}{$22.0-45.0$} \\
Mean \pm SD. & \multicolumn{2}{|c|}{$30.38 \pm 5.14$} \\
Median & \multicolumn{2}{|c|}{30.0} \\
\hline
\end{tabular}

Table (2): Distribution of the studied cases according to complaint $(\mathbf{n}=\mathbf{1 0 0})$

\begin{tabular}{|c|c|c|}
\hline Complaint & No. & \% \\
\hline Bleeding & 39 & 39.0 \\
Infertility & 61 & 61.0 \\
\hline
\end{tabular}

Table (3): Comparison between hysteroscopy findings and 3D US findings $(\mathbf{n}=100)$

\begin{tabular}{|c|c|c|c|c|}
\hline \multirow{2}{*}{} & \multicolumn{2}{|c|}{$\begin{array}{c}\text { 3D US } \\
\text { findings }\end{array}$} & \multicolumn{2}{c|}{$\begin{array}{c}\text { Hysteroscopy } \\
\text { findings }\end{array}$} \\
\cline { 2 - 5 } & No. & \% & No. & \% \\
\hline Free & 15 & 15.0 & 0 & 0.0 \\
Positive & $\mathbf{8 5}$ & $\mathbf{8 5 . 0}$ & $\mathbf{1 0 0}$ & $\mathbf{1 0 0 . 0}$ \\
\hline Polyp & 40 & 40.0 & 50 & 50.0 \\
Adhesions & 5 & 5.0 & 8 & 8.0 \\
Myoma & 13 & 13.0 & 13 & 13.0 \\
Septum & 27 & 27.0 & 29 & 29.0 \\
\hline Accuracy & $\mathbf{8 5}$ & $\mathbf{8 5 . 0}$ & $\mathbf{1 0 0}$ & $\mathbf{1 0 0 . 0}$ \\
\hline
\end{tabular}

Table (4): Sensitivity, specificity, positive predictive value, negative predictive value and accuracy for Sonography compared definitive diagnosis

\begin{tabular}{||c|c|c|c|c|c|}
\hline $\begin{array}{c}\text { Pathologica } \\
\text { Ifinding }\end{array}$ & Sensitivity & Specificity & PPV & NPV & Accuracy \\
\hline Normal & $74.2 \%$ & $84.47 \%$ & $52.1 \%$ & $72.86 \%$ & $86 \%$ \\
\hline Polyp & $67.2 \%$ & $88.3 \%$ & $63.1 \%$ & $91.6 \%$ & $90 \%$ \\
\hline Septate & $95.7 \%$ & $94.1 \%$ & $84.1 \%$ & $94.2 \%$ & $91.3 \%$ \\
\hline $\begin{array}{c}\text { Submucous } \\
\text { fibroid }\end{array}$ & $100 \%$ & $100 \%$ & $100 \%$ & $100 \%$ & $100 \%$ \\
\hline $\begin{array}{c}\text { Intrauterin } \\
\text { eadhesions }\end{array}$ & $65.2 \%$ & $83 \%$ & $81 \%$ & $67.3 \%$ & $77.8 \%$ \\
\hline
\end{tabular}

Discussion:

Uterine cavity is an important factor for successful implantation of the embryo.
Presence of benign intrauterine lesion, such as endometrial polyps, uterine septum, intrauterine adhesions and submucous myoma may contribute to infertility or decrease chances of successful ART or bleeding.

Therefore, accurate and reliable tools for uterine cavity assessment are very important. Our study showed the diagnostic accuracy of 3D-TVS to be $84.1 \%$. 3D-TVS showed sensitivity of $68.2 \%$, specificity of $91.5 \%$, positive predictive value of $79 \%$, and negative predictive value of $86 \%$.

We reviewed previous studies regarding diagnostic accuracy of 3D-TVS for detecting uterine cavity lesion in infertility patients and found no matching studies. A study by Van den Bosch $^{(9)}$ evaluated diagnostic accuracy of 3DTVS in detection of uterine cavity lesion in women with abnormal uterine bleeding. They found endometrial polyp in $26 \%$, submucous myoma in $7 \%$, endometrial hyperplasia in $6 \%$, and cancer in $1 \%$ of subjects. They also reported 93\% diagnostic accuracy, $96 \%$ sensitivity, and $91 \%$ specificity.

Our results showed lower diagnostic accuracy than the Van den Bosch study. Differences in pathology and size of lesion affected these outcomes. In our study, 3D-TVS detected all cases of submucous myoma while missed two cases out of twenty nine cases with septate uterus. Even though there were only a small number of cases with submucous myoma, 3DTVS demonstrated $100 \%$ diagnostic accuracy. For detection of endometrial polyp, our study showed $80 \%$ sensitivity, $100 \%$ specificity, $90 \%$ diagnostic accuracy, $100 \%$ positive predictive value, and $83.33 \%$ negative predictive value. Our results were similar to results from a study by Fang ${ }^{(2)}$ that reported sensitivity of $65.6 \%$ and specificity of $89 \%$ for 3D-TVS in detection of endometrial polyps using combined parameters of endometrial echogenicity, endometrial thickness, and endometrial volume. In our study, 3D-TVS failed to detect ten endometrial polyps, all of which had diameter $8 \mathrm{~mm})^{\left({ }^{(10)}\right.}$. Uterine cavity lesion is an important and correctable factor that can lead to improvement inART outcome or a condition of abnormal uterine bleeding.

Identification of accurate and reliable detection and diagnostic tools for assessment of the uterine cavity is a necessary first step in the improvement of ART success rates. 3D-TVS had $84.1 \%$ diagnostic accuracy for detecting uterine cavity abnormalities in infertile women. 
Our study demonstrated that 3D TVS had high specificity, but its sensitivity was limited, especially for detecting endometrial polyp. A significant percentage of infertile patients had evidence of uterine cavity pathology. As such, hysteroscopy remains the preferred procedure for accurate detection and diagnosis of uterine cavity lesion.

\section{Conclusion:}

Hysteroscopy is recommended for accurate detection and diagnosis of uterine cavity lesion.

\section{References:}

1. Pundir J, El Toukhy T (2010): Uterine cavity assessment prior to IVF. Womens Health (Lond), 6(6):841-7.

2. Fang L, Su Y, Guo Y, Sun Y (2013): Value of 3-dimensional and power Doppler sonography for diagnosis of endometrial polyps. J Ultrasound Med., 32(2):247-55.

3. Doldi N, Persico P, Di Sebastiano F, Marsiglio E, De Santis L, Rabellotti E $e t$ al. (2005): Pathologic findings in hysteroscopy before in vitro fertilizationembryo transfer (IVF-ET). Gynecol Endocrinol., 21(4):235-7.

4. Bahadur A, Malhotra N, Singh N, Gurunath S, Mittal S (2013): Comparative study on the role of diagnostic hysteroscopy in evaluation of the uterine cavity prior to in vitro fertilization in a developing country. Arch Gynecol Obstet., 288(5):1137-43.

5. Downey DB, Fenster A, Williams JC (2000): Clinical utility of threedimensional US. Radiographics , 20(2):559-71.
6. Vercellini P, Cortesi I, Oldani S, Moschetta M, De Giorgi O, Crosignani PG (1997): The role of transvaginal ultrasonography and outpatient diagnostic hysteroscopy in the evaluation of patients with menorrhagia. Hum Reprod., 12(8):1768-71.

7. Society for Assisted Reproductive Technology; American Society for Reproductive Medicine (2007): Assisted reproductive technology in the United States: 2001 results generated from the American Society for Reproductive Medicine/Society for Assisted Reproductive Technology registry. Fertil Steril., 87(6):1253-66.

8. Michael SB, Rafael FV, Hubert G (2007): Hysteroscopy: visual perspectives of uterine anatomy, physiology, and pathology. $3^{\text {rd }}$ ed. Philadelphia: Lippincott Williams \& Wilkins.

9. Van den Bosch T, Valentin L, Van Schoubroeck D, Luts J, Bignardi T, Condous G et al. (2012): Detection of intracavitary uterine pathology using offline analysis of three-dimensional ultrasound volumes: interobserver agreement and diagnostic accuracy. Ultrasound Obstet Gynecol., 40(4):45963.

10. Rackow BW, Jorgensen E, Taylor HS (2011): Endometrial polyps affect uterine receptivity. Fertil Steril., 95(8):2690-2. 